\title{
Tecnura
}

http://revistas.udistrital.edu.co/ojs/index.php/Tecnura/issue/view/640

\section{Prototipo de sistema experto en diagnóstico médico basado en síntomas de los pacientes. Caso de estudio: esclerosis múltiple}

\author{
Expert system prototype on medical diagnosis based on patients' symptoms. \\ Case study: multiple sclerosis
}

\author{
Juan Guillermo Rivera Berrío*, Héctor Aníbal Tabares Ospina**
}

Citation / Para citar este artículo: Rivera Berrio, J. G., \& Tabares Ospina, H. A. (2014). Prototipo de sistema experto en diagnóstico médico basado en síntomas de los pacientes. Caso de estudio: esclerosis múltiple. Revista Tecnura, Edición especial, 215-216.

Fecha de recepción: 16 de julio de 2013 / Fecha de aceptación: 7 de marzo de 2014

\begin{abstract}
Resumen
Se presenta en este artículo un modelo de sistema experto para el diagnóstico de la esclerosis múltiple. Esta labor no es una tarea trivial, debido a la subjetividad que puede presentarse en su evaluación. Este proceso se puede complementar usando un sistema de apoyo a la toma de decisiones. El sistema desarrollado se dividió en cuatro fases: toma de requisitos, diseño, implementación y puesta en marcha. Con el prototipo software se logró modelar el conocimiento específico del experto neurólogo, lo que permitió obtener un diagnóstico de la esclerosis múltiple.
\end{abstract}

Palabras clave: sistema experto, esclerosis múltiple.

\begin{abstract}
This paper presents a model of expert system for the diagnosis of multiple sclerosis. This task is not a trivial task, due to the subjectivity that may occur in their evaluation. This process can be supplemented using a system to support decision making. The developed system was divided into four phases: making requirements, design and implementation, and its set-up. With the software prototype, it was possible to model the specific knowledge of the expert neurologist, allowing a diagnosis of multiple sclerosis.
\end{abstract}

Keywords: Expert system, multiple sclerosis.

\footnotetext{
* Ingeniero civil, especialista en estructuras, doctor en estudios de ciencia y tecnología. Vicerrector académico, Institución Universitaria Pascual Bravo. Contacto jg.rivera@pascualbravo.edu.co

** Ingeniero electricista, especialista en ingeniería del software, magíster en ingeniería de sistemas, estudiante de doctorado en sistemas energéticos. Docente, Institución Universitaria Pascual Bravo. Medellín, Colombia. Contacto: h.tabares@pascualbravo.edu.co
} 


\section{INTRODUCCIÓN}

El objetivo fundamental de este artículo consiste en hacer un desarrollo software para detectar la esclerosis múltiple, EM. Su diagnóstico ha sido una de las constantes preocupaciones de los expertos en el tema, debido a la dificultad que existe en formalizar adecuadamente los síntomas. Para su diagnóstico, la subjetividad está presente como una característica permanente, lo que llevó para su solución, al desarrollo de un sistema experto -SE- del tipo determinista, titulado SistemaExperto_IUPB, en el cual se confirmó que los SE brindan grandes posibilidades en el campo clínico.

Este trabajo comienza con el estado del arte de los SE usados para el diagnóstico de enfermedades. A continuación se presenta la metodología utilizada para la implementación de este sistema. Continúa el artículo mostrando las pruebas de validación y los resultados más importantes. Finaliza presentando las principales conclusiones del trabajo investigativo y el esbozo de trabajos futuros.

\section{Los sistemas expertos}

Un SE es una aplicación informática que, sobre una base de conocimientos -BC-, posee información de uno o más expertos para solucionar un conjunto de problemas en un área específica. La BC es un tipo especial de bases de datos -BDpara la gestión del conocimiento, que posee una considerable capacidad de deducción a partir de la información que contiene. La diferencia entre la BD y la BC consiste en que la primera almacena únicamente hechos (afirmaciones que sirven para representar conceptos, datos, objetos, etc.) y las funciones del motor de la BD son las de edición y consulta de los datos. La segunda, por otra parte, puede almacenar, además de hechos (base de hechos que describen un proble$\mathrm{ma}$ ), un conjunto de reglas. Una regla es una estructura condicional que relaciona lógicamente la información contenida en la parte del antecedente con otra información contenida en la parte del consecuente. En una BC, las reglas se sirven de esos hechos para que el motor de inferencia MI- obtenga razonamiento deductivo automático, seleccionando las reglas posibles para solucionar un determinado problema y así conseguir información que no se encuentra almacenada en forma explícita.

Tanto con la BD como con la BC se pueden realizar consultas dinámicas. En el primer caso, es la posibilidad que tiene el usuario final para configurar la consulta en tiempo de ejecución. En el segundo caso, el usuario introduce la información del problema actual en la base de hechos y el sistema empareja esta información con el conocimiento disponible en la base de conocimientos para deducir nuevos hechos.

Los SE pueden clasificarse en dos tipos principales, según la naturaleza de los problemas para los que están diseñados: deterministas y estocásticos. Los SE que tratan problemas deterministas se conocen como "sistemas basados en reglas", porque sacan sus conclusiones basándose en un conjunto de reglas utilizando un mecanismo de razonamiento lógico.

Los sistemas que tratan problemas estocásticos, es decir, problemas que involucran situaciones inciertas, necesitan introducir medios para medir la incertidumbre. Algunas medidas de incertidumbre son los "factores de certeza" y la "probabilidad". Estos SE utilizan una distribución de probabilidad conjunta de un grupo de variables para describir las relaciones de dependencia entre ellas y así sacar conclusiones usando fórmulas de la teoría de probabilidad. Para una lograr definición más eficiente de la distribución de probabilidad conjunta se utilizan modelos de redes probabilísticas, entre los que se incluyen las redes de Markov y las redes Bayesianas.

Un detallado estudio sobre SE está más allá del ámbito de esta sección. En (Giarratano \& Riley, 2001) se ofrece un excelente estudio con referencias específicas. 


\section{Antecedentes}

La EM es una enfermedad desmielinizante, neurodegenerativa y crónica del sistema nervioso central. En su génesis actúan mecanismos autoinmunes y a causa de sus efectos, puede tener como consecuencia una movilidad reducida e invalidez en los casos más severos. Después de la epilepsia, es la enfermedad neurológica más frecuente entre los adultos jóvenes desde la erradicación de la poliomielitis, y es la causa más frecuente de parálisis en los países occidentales (Gutierrez, Gonzalez, Lopez, Ojeda, Sanchez, \& Toro, 2001). En (AMERICAN ACADEMIC OF NEUROLOGY, 2011) se informa que la EM afecta aproximadamente a 1 de cada 1.000 personas con edades entre 20 y 40 años, más comúnmente en las mujeres.

Con el propósito de estudiar la evolución clínica de la enfermedad, se han empezado a usar nuevas herramientas informáticas inteligentes. En (Shin, Tolum, \& Hassanpour, 2012) se presenta un análisis estadístico de los enfoques de sistemas expertos aplicados en medicina, en el que se indica un aumento en el número de publicaciones sobre sistemas interconectados con redes neuronales artificiales RNA- y neurodifusos-ND-. Estos últimos combinan las técnicas de las RNA y las técnicas de inferencia borrosa. La lógica borrosa proporciona un mecanismo de inferencia sobre la incertidumbre y las redes neuronales ofrecen las ventajas computacionales en cuanto al aprendizaje y la generalización.

Así las cosas, un sistema neuronal artificial fue aplicado en (Chiou \& Lure, 1994) para detectar células que producen cáncer de pulmón. A partir de este estudio surgieron otros trabajos en reconocimiento de imágenes en radiología, dermatología, patología, endoscopia (Xu, Brown, Breit, \& Nakamura, 2006), análisis de rayos $X$ aplicados en exámenes de mamografía y radiografías de pecho (Wang \& Shiun, 2009).

Dentro de la literatura médica existen numerosos estudios que emplean modelos ND. En (Celona, Grasso, \& Puccio, 2009) realizaron una clasificación morfológica de imágenes obtenidas con exámenes de resonancia magnética. Consiste en procesar las imágenes usando una RNA para aumentar su tasa de reconocimiento. Como resultado final, se presenta una RNA capaz de distinguir los diferentes contornos de plaquetas producidas por la EM.

Otros estudios evaluaron la aplicación de sistemas ND para el diagnóstico de infarto del miocardio (Buarque, 2000), (Bizios, Heij, \& Bengtsson, 2007), artritis celular (Astion, Wener, Thomas, Hunder, \& Bloch, 1994), (Borges, Moura, \& Steiner, 2010), tipos de cáncer (Bourdes, Bonnevay, Bachelot, \& Perol, 2007), pronosticar la duración de pacientes en la unidad de cuidados intensivos (Cook, 2005), evolución de enfermedades después de realizarse una operación de trasplante (Doyle, y otros, 1994), (Gil, Johnsson, Garcia, \& Soriano, 2009). Otros estudios que usan RNA están relacionados con enfermedades cardio-pulmonares y grado de sobreviviencia después del trauma (EbeII, 1993), (Harrison \& Kennedy, 2005), interpretación de pruebas de diagnóstico relacionadas con el páncreas (Kasmierczak, Catrou, \& Van Lente, 1998), (Bartosh, Andersson, \& Nilsson, 2008) y tiroideos (Sharpe, Solberg, Rootwelt, \& Yearworth, 1993), (Gannous \& Elhaddad, 2011).

Con respecto a la EM, varios ensayos experimentales sobre terapia están en curso, con el fin de descubrir un tratamiento exitoso. La mayoría de estos estudios de investigación utilizan una escala de calificación clínica denominada Escala Ampliada del Estado de Discapacidad (EAED) como herramienta de evaluación de los efectos de las drogas. Esta escala se define por un conjunto de reglas que proporcionan una cuantificación numérica de la exploración neurológica. Aunque EAED ha sido ampliamente utilizado durante casi 20 años, su aplicación sigue dependiendo de la interpretación del neurólogo, por lo que la evaluación efectuada por diferentes neurólogos en el mismo paciente puede dar resultados diferentes. Este es un problema grave para los ensayos internacionales debido a que carecen de una medida fiable de los efectos de las drogas. En (Gaspari, Roveda, Scandellari, \& Stecchi, 2012) se presenta un sistema experto para 
la evaluación automática de EAED en la EM. El sistema experto es una herramienta de evaluación complementaria capaz de apoyar al usuario para hacer una representación explícita de reglas EAED.

El uso de la resonancia magnética como una herramienta de apoyo en el diagnóstico y seguimiento de la EM y en la evaluación de los efectos del tratamiento, requiere la determinación precisa de la lesión cerebral que produjo la enfermedad. Para apoyar de manera automática a los neurorradiólogos en la clasificación de la lesión cerebral, en (Esposito \& De Pietro, 2011) se desarrolló un sistema experto para apoyar las decisiones difusas basadas en ontologías(DSS). El DSS codifica el conocimiento médico de alto nivel, especializado en términos de ontologías y reglas difusas, y aplica este conocimiento en conjunción con un motor de inferencia borrosa para clasificar la lesión cerebral. El rendimiento de la DSS ha sido evaluado cuantitativamente en120 pacientes afectados por EM. Un análisis estadístico se ha llevado a cabo para evaluar la influencia de DSS en las tareas de diagnóstico de los médicos. La evaluación ha demostrado que el DSS ofrece una forma innovadora y valiosa para llevar a cabo la clasificación de las lesiones cerebrales, en forma automatizada y en entornos clínicos reales.

A pesar de lo anterior, es un reto implementar en software una evaluación global para el diagnóstico de la EM. Esta es una razón por la cual en la INSTITUCIÓN UNIVERSITARIA PASCUAL BRAVO-IUPB-se ha trabajado en el diseño de modelos computacionales usando SE, como el expuesto en (Primorac \& Mariño, 2010), (Tabares, Monsalve, \& Diez, 2013) desarrollado para asistir decisiones turísticas, tomado y adaptado para los propósitos de este trabajo investigativo. Se pretende superar las limitaciones de los previos modelos expuestos, referidos a la exactitud y precisión de los resultados finales.

\section{MATERIALES Y MÉTODOS}

Los actores que intervienen en el aplicativo SistemaExperto_IUPB son: el ingeniero de sistemas, que en este caso hace las veces de ingeniero del conocimiento -IC-, el experto en el dominio de conocimiento -EDC- sobre esclerosis múltipleEM- y los usuarios finales -UF- que interactúan con el sistema con miras a determinar si un paciente tiene EM.

La metodología abordada para la implementación del SE contempló las siguientes etapas:

- Identificación del problema, en este caso, la determinación de la enfermedad EM.

- Selección de la variable objetivo y sus valores. El EDC definió como variable objetivo el diagnóstico de la enfermedad EM.

- La selección de las variables de entrada y sus posibles valores: sobre la base del conocimiento del EDC, se seleccionaron el conjunto de variables de entrada relevantes. En el modelo abordado en este trabajo, las preferencias están determinadas por los indicadores básicos de síntomas de que se detallan en la Tabla 1. Debido a que los nervios en cualquier parte del cerebro o la médula espinal pueden resultar dañados, los pacientes

Tabla 1. Indicadores variables de entrada (síntomas básicos).

\section{Síntomas musculares}

Pérdida del equilibrio

Espasmos musculares

Entumecimiento o sensación anormal en cualquier área

Problemas para mover los brazos y las piernas

Problemas para caminar

Problemas con la coordinación y para hacer movimientos pequeños

Temblor en uno o ambos brazos o piernas

Debilidad en uno o ambos brazos o piernas

\section{Síntomas intestinales}

Estreñimiento y escape de heces

Dificultad para comenzar a orinar

Necesidad frecuente de orinar

Urgencia intensa de orinar

Escape de orina (incontinencia)

\section{Síntomas oculares}

Visión doble

Movimientos oculares rápidos e incontrolables

Pérdida de visión (usualmente afecta un ojo cada vez) 
con EM pueden tener síntomas en muchas partes del cuerpo. Por tanto, su clasificación es muy variada, por lo que, para los propósitos de este artículo, se listan los más destacados según sus efectos a nivel muscular, intestinal u ocular.

Si se activan cualesquiera de los síntomas a nivel muscular, intestinal u ocular, entonces se infiere la EM.

Los síntomas listados no constituyen la universalidad de casos, por lo que el programa SistemaExperto_IUPB provee los medios para la recolección de nuevos síntomas.

Es importante recalcar que los síntomas antes listados no siempre son indicios de EM, dado que pueden deberse de igual manera a otros trastornos neurológicos. Así mismo, el hecho de presentar uno o dos de estos síntomas en forma aislada tampoco significa necesariamente la EM. Por lo general, para considerarla se requieren tres o más de dichos síntomas presentados en forma conjunta.

Además del análisis de los síntomas, el médico puede sospechar de EM si hay disminución en el funcionamiento de dos partes diferentes del sistema nervioso central (como los reflejos anormales) en dos momentos diferentes. En este caso, se efectúa un examen neurológico como el presentado en la Tabla 2. Las pruebas de funciones neurológicas pueden mostrar disminución en la función nerviosa en un área del cuerpo o diseminación sobre muchas partes del cuerpo.

Tabla 2. Exámenes neurológicos.

\section{Pruebas funciones neurológicas}

Reflejos nerviosos anormales.

Sensibilidad anormal o disminuida.

Disminución capacidad motora.

Respuestas anormales de la pupila.

Cambios en los campos visuales o en los movimientos oculares.

Movimientos oculares rápidos provocados por movimiento del ojo.
Los exámenes clínicos realizados para estudiar el estado y progresión de la EM abarcan los listados en la tabla 3.

Tabla 3. Exámenes para el diagnóstico.

\section{Exámenes para el diagnóstico de la EM}

Punción lumbar.

Resonancia magnética del cerebro y la columna.

Estudio de la función neurológica (potenciales provocados).

Hasta el momento, no se conoce cura para la EM; sin embargo, existen terapias que pueden retardar el progreso de la enfermedad. El objetivo del tratamiento es controlar los síntomas y ayudar a mantener una calidad de vida normal.

Los medicamentos utilizados para disminuir la progresión de la EM se toman durante un tiempo prolongado y pueden abarcar, de manera genérica, los que se presentan en la Tabla 4.

El pronóstico de la evolución de la enfermedad es variable y difícil de predecir. Aunque el trastorno es crónico e incurable, la expectativa de vida puede ser normal o casi normal. La mayoría de las personas con EM continúan caminando y desempeñándose en el trabajo con discapacidad mínima durante 20 años o más.

- Diseño de las preguntas. El desarrollo del cuestionario es la parte más complicada y en general es una actividad iterativa. El proceso se puede comenzar identificando los rasgos y características, representadas en las variables de entrada, presentes o no en los valores que asume la variable objetivo identificada para el dominio del problema.

Tabla 4. Tratamiento.

\begin{tabular}{l}
\hline \multicolumn{1}{c}{ Tratamiento } \\
\hline Interferón. \\
Esteroides (utilizados para disminuir la gravedad de los \\
ataques). \\
Lioresal (para disminuir los espasmos musculares) \\
Medicamentos colinérgicos (para disminuir los problemas \\
urinarios)
\end{tabular}


En esta fase intervinieron el IC y el EDC. La adquisición de la información relevante se obtuvo consultando con el EDC y el IC extrajo las principales características de la EM. Con base en los rasgos identificados en el paso anterior, el IC y el EDC diseñan las reglas por evaluar y las preguntas por formular a los usuarios finales -UF- del sistema cuando interactúa con el subsistema de interfaz de UFI. En la Tabla 5 se presentan las reglas por evaluar.

El SE intercambia datos entre las tablas 1, 2, 3, 4 y 5 para inferir la EM. La clasificación de la EM es muy variada, agrupándose por los síntomas -muscular, intestinal u ocular-.

Implementación software. En las especificaciones de diseño del aplicativo SistemaExperto_IUPB se manejaron tres capas: interfaz, lógica de programa, datos; esto para lograr que la interfaz sea completamente independiente del sistema y la BC pueda alterarse sin tener que hacer cambios en la programación. La BC reside en un manejador de bases de datos. La base de reglas y el $\mathrm{Ml}$ se implementaron en lenguaje C\# para ambiente de escritorio, utilizando el escenario de desconectado para tener acceso al motor de la BD.

La arquitectura básica del aplicativo SistemaExperto_IUPB se puede observar en la figura 1.

El programa SistemaExperto_IUPB está compuesto por tres subsistemas: i) el editor de variables de entrada y salida, ii) la base de conocimiento, iii) la interfaz de usuario final.

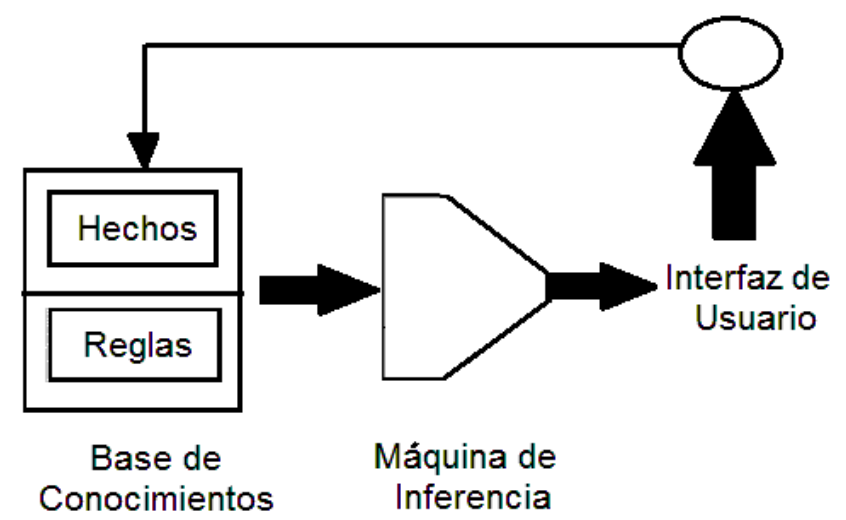

Figura 1. Arquitectura del aplicativo Sistema Experto_IUPB.

\section{Editor de variables de entrada y salida}

Con base en los rasgos identificados en las variables de entrada, el IC y el EDC las registran utilizando el subsistema editor de variables de entrada (antecedentes) y salida (objetivos o consecuentes). En la figura 2 se ilustran algunas formulaciones que estarán disponibles.

El control combo titulado "Tipo Variable" permite ingresar una nueva variable del tipo antecedente o consecuente.

\section{Editor base de conocimiento}

El archivo de las variables de entrada y salida se carga en el subsistema de adquisición del conocimiento. Inicialmente el sistema no contiene posibles

Tabla 5. Subclases.

\begin{tabular}{cll}
\hline No & \multicolumn{1}{c}{ Regla antecedente (daños o efectos) } & \multicolumn{1}{c}{ Consecuente } \\
\hline 1 & Síntomas a nivel muscular, intestinal u ocular & Síntomas de EM \\
\hline 2 & Síntomas de EM & Efectuar pruebas de función neurológica (PFN) \\
\hline 3 & PFN de reflejos o disminución de capacidad motora. & Diagnóstico EM \\
\hline 4 & Diagnóstico EM. & $\begin{array}{l}\text { Realizar exámenes punción lumbar, RM cerebral y colum- } \\
\text { na, potenciales evocados. }\end{array}$ \\
\hline 5 & $\begin{array}{l}\text { Si exámenes punción lumbar, RM cerebral y columna, } \\
\text { potenciales evocados confirman diagnóstico de EM. }\end{array}$ & Tratamiento con interferones y esteroides. \\
\hline
\end{tabular}




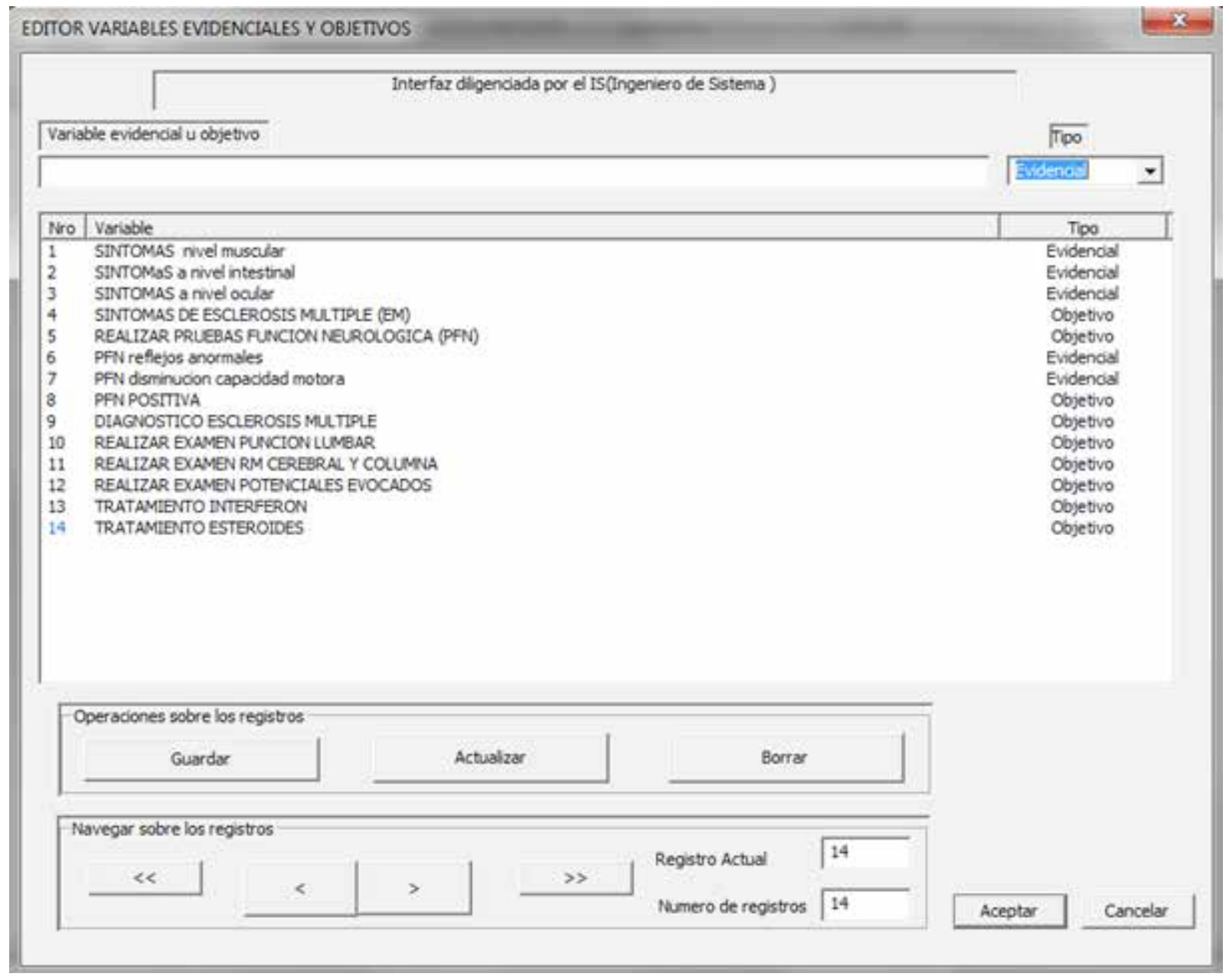

Figura 2. Editor de variables de entrada y salida. El IC implementa el editor y el EDC lo diligencia en tiempo de ejecución.

reglas. Como se presenta en la figura 3, el EDC carga las variables generadas por el IC y configura las reglas en la base de conocimiento. Este proceso se repite hasta que se hayan configurado todas las soluciones posibles para cada una de las preguntas formuladas.

Es frecuente que diferentes sucesos estén correlacionados, siendo necesario hacer cierta pregunta si la respuesta seleccionada a la pregunta anterior lo requiere. El subsistema permite establecer dependencias por medio de su opción "Crear Dependencia".

Finalizado este proceso, las preguntas formuladas, las dependencias y la importancia asignada se guardan en una base de datos.

\section{Bases de datos}

Todo el sistema está relacionado como una base de datos Access (MDB) titulada SistemaExperto-EM_IUPB.db, y centra su gestión en tres aspectos principales: síntomas, clasificación (muscular, intestinal u ocular), exámenes neurológicos, diagnóstico y tratamiento. El esquema de la base de datos relacional que cumple con el planteamiento de los requisitos se presenta en la figura 4. El significado de las tablas y sus relaciones es el siguiente:

- Tbl_Sintomas. Representa los síntomas. 


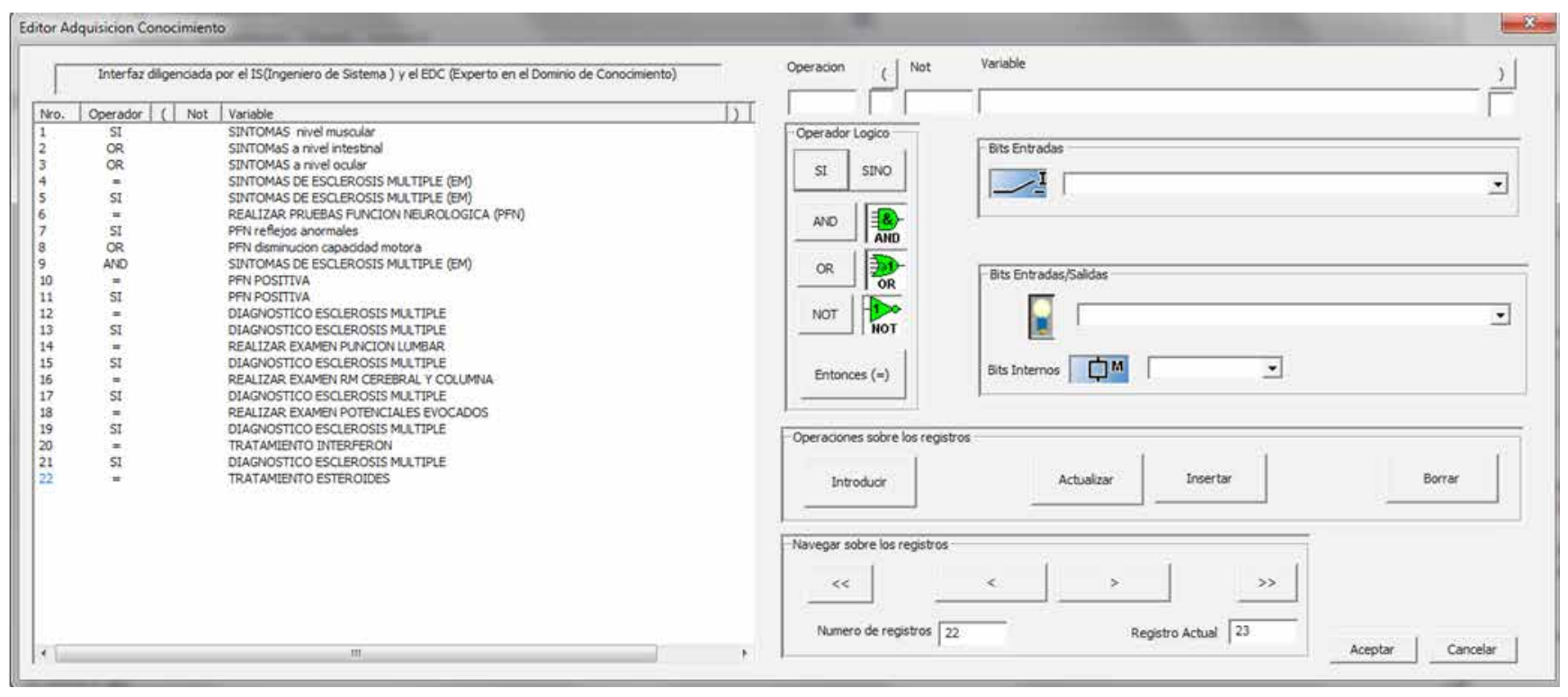

Figura 3. Editor adquisición del conocimiento. Diligenciado en tiempo de ejecución por el EDC.
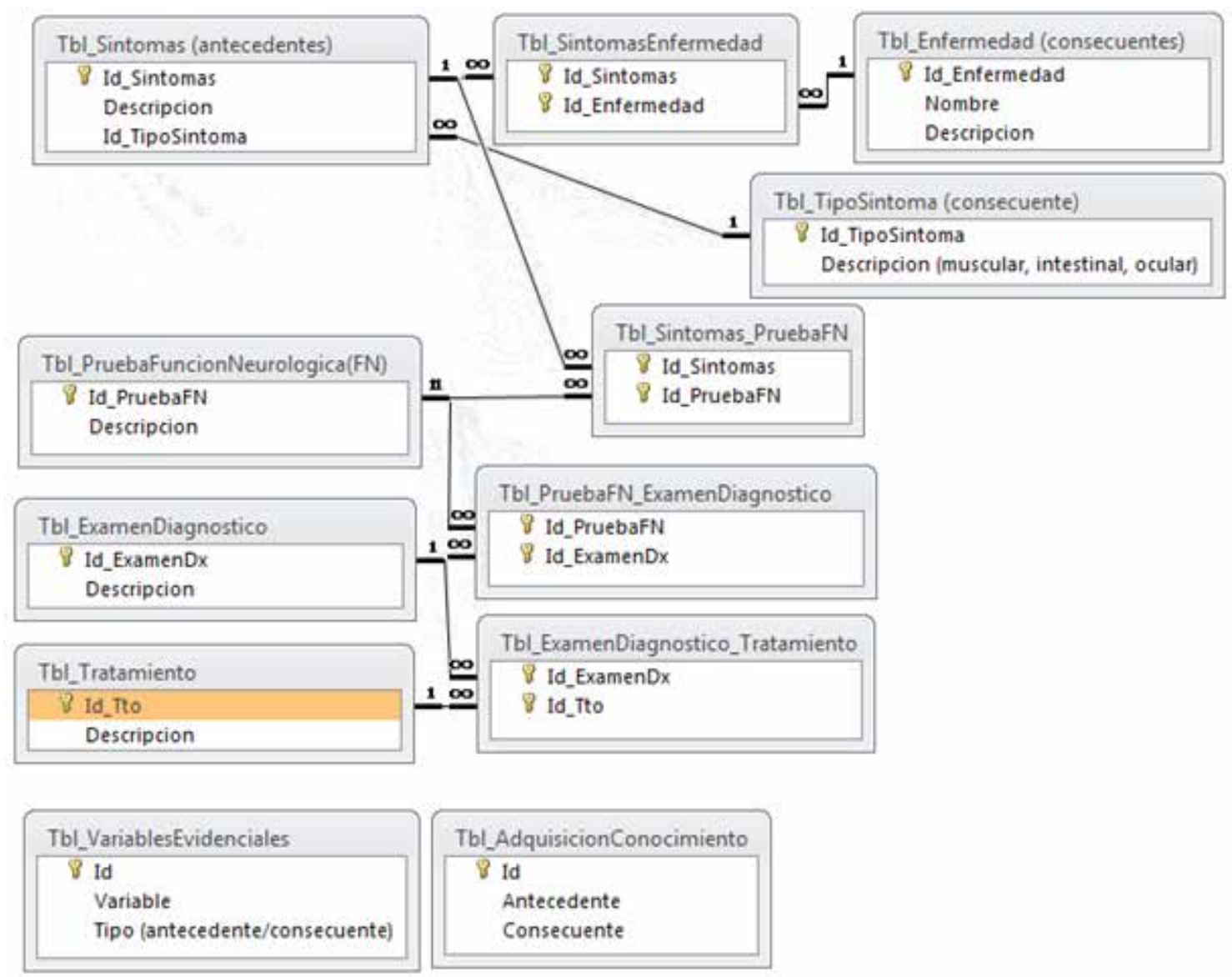

Figura 4. Diagrama entidad-relación de la BC del SE. 
- Tbl_TipoSintoma, indica la clasificación de los síntomas según su afectación a nivel muscular, intestinal u ocular.

- Tbl_Enfermedad, contiene el listado de enfermedades.

- Tbl_VariablesEvidenciales, variables del tipo entrada o salida del SE.

- Tbl_AdquisicionConocimento, comprende el listado de reglas que evalúa el SE.

El significado de los atributos que componen las diferentes tablas es explícito, de tal manera que los lazos entre las relaciones son fáciles de entender. Por ejemplo, el lazo que conecta Tbl_Sintomas (Antecedentes) con Tbl_TipoSintoma (Consecuentes) sirve para clasificar el síntoma a nivel muscular, intestinal u ocular.

La relación entre Tbl_Sintomas (Antecedentes) y Tbl_Enfermedad (Consecuentes), al tratarse de una relación con cardinalidad VARIOS a VARIOS, no está permitida por cuanto contradice la definición de llave primaria y las reglas de normalización de bases de datos. En estos casos, se hace necesario crear una nueva tabla que permita transformar las relaciones en cardinalidades UNO a VARIOS. De ahí que se haya creado la entidad relación Tbl_SintomasEnfermedad. En este caso, un síntoma puede estar presente en muchas enfermedades y una enfermedad puede presentar muchos síntomas.

\section{Interfaz de usuario final}

La figura 5 ilustra el subsistema de interacción con el UF. El subsistema de interacción con el UF permite el acceso al conocimiento especializado, que puede consultarse interactivamente en cada paso, luego de seleccionar la variable de entrada y su valor en el recorrido desde el inicio a la meta.

El cuestionario primero se presenta al EDC cuando utiliza el subsistema de adquisición del conocimiento para completar la BC y verificar cómo están relacionadas cada una de las opciones disponibles para recomendar una solución.

El funcionamiento general del subsistema de interacción con el UF es el siguiente: el UF selecciona el conjunto de variables evidenciables relevantes sobre los síntomas, y responde las preguntas planteadas por el SE. En el modelo abordado en este trabajo, las preferencias están determinadas por las variables: i) síntomas, ii) clasificación.

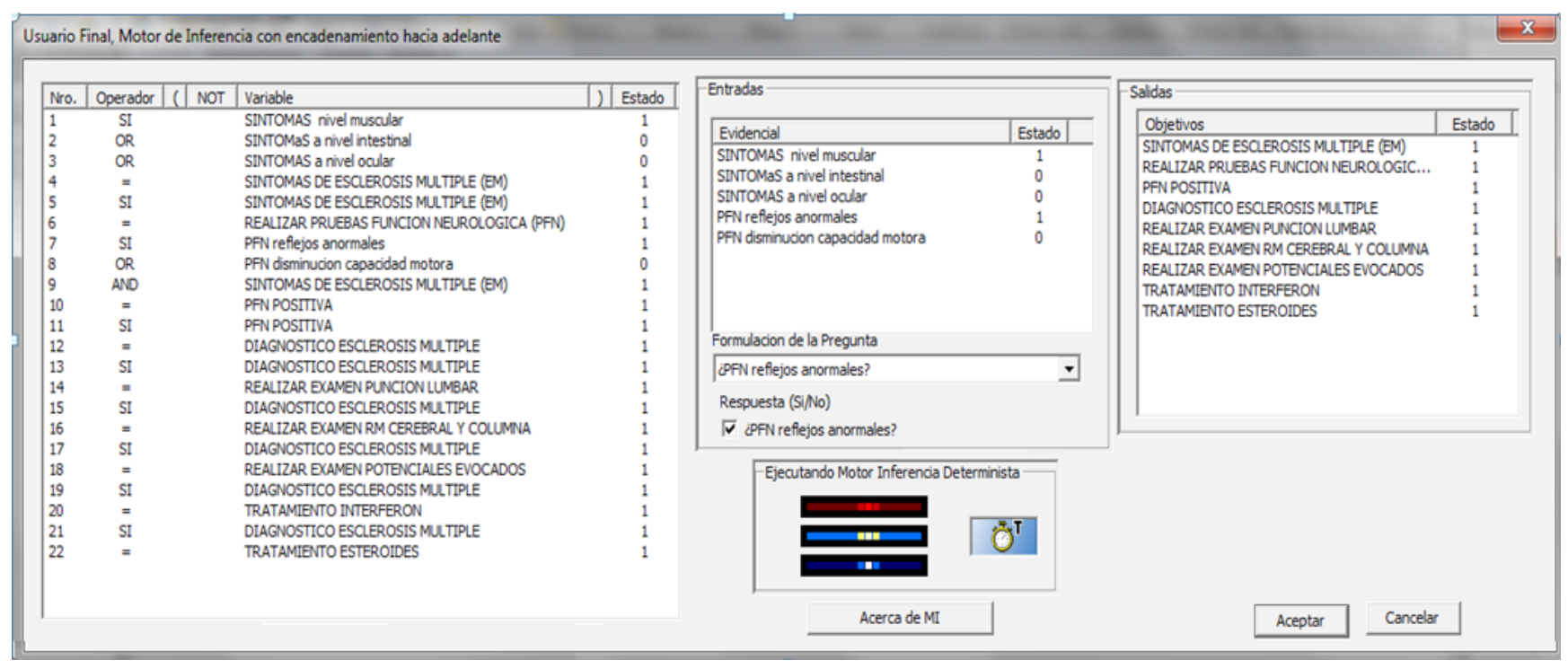

Figura 5. Interfaz UF. 
La interfaz que maneja la interacción entre el SE y el UF es altamente interactiva y sigue el patrón de la conversación a nivel de escritura entre seres humanos. Por tanto, un requerimiento básico de la interfaz es la habilidad de hacer preguntas. Para obtener información fiable del usuario, es necesario prestar especial cuidado en el diseño del cuestionario. Esto requirió diseñar la interfaz usando menús o gráficos.

La consulta transcurre en general según el esquema siguiente: primero se plantea al usuario la pregunta sobre los síntomas para alcanzar una determinación aproximada del contexto. El diálogo, por parte del sistema, está a menudo dimensionado para ir confirmando o rechazando hipótesis (por ejemplo, comprobación de la presencia de EM), o para realizar una aproximación sucesiva hacia un objetivo introducido de antemano (por ejemplo, clasificación).

Una vez finalizado el diálogo, el componente explicativo suministra, si es necesario, el historial completo de la consulta.

La configuración efectuada por el UF es empleada por el MI para obtener razonamiento deductivo automático, seleccionando las reglas posibles para especificar el tipo de EM.

\section{RESULTADOS}

Para probar el sistema, se empleó el subsistema de interfaz de interacción con el UF. Con los datos definidos en los filtros, el sistema SistemaExperto_IUPB simula al experto humano, usando el MI, aplicando un encadenamiento de reglas hacia adelante para contrastar los hechos particulares de la base de hechos con el conocimiento contenido en la BC y obtener conclusiones acerca de la consulta realizada. El algoritmo consiste en asignar a los objetos sus valores conocidos como lo dan los hechos o evidencias. Seguidamente se ejecuta cada regla de la base de conocimiento y se concluye nuevos hechos si es posible. Finalmente, se repite el paso anterior hasta que no puedan obtenerse nuevos hechos. Para la evaluación de cada regla, el MI la convierte de notación infijo a posfijo. Seguidamente, el MI aplica una subrutina evaluadora de expresiones en notación posfijo. En la tabla 3 se presenta la inferencia del SE ante 3 posibles síntomas.

\section{CONCLUSIONES}

El objetivo de este trabajo consistió en desarroIlar el prototipo software SistemaExperto_IUPB orientado a diagnosticar la esclerosis múltiple. EI programa se consultará ante todo cuando existan síntomas de la enfermedad que no requieren tratamiento inmediato y no se pueda o quiera recurrir primero al experto humano. Asimismo, el aplicativo sirve también al mismo experto que lo alimenta como diccionario dinámico, que mantiene el conocimiento de reglas antiguas, mientras se concentran en los nuevos desarrollos.

El prototipo de SE usando un MI para obtener razonamiento deductivo automático permite

Tabla 3. Operación del SE.

\begin{tabular}{ll}
\hline \multicolumn{1}{c}{$\begin{array}{c}\text { VARIABLES DE ENTRADA } \\
\text { ANTECEDENTE }\end{array}$} & \multicolumn{1}{c}{ VASTEMA EXPERTO EM } \\
\hline \multicolumn{1}{c}{ VARIABLES DE SALIDA ACTIVADAS POR EL MI } \\
CONSECUENTE
\end{tabular}


obtener una recomendación sobre el tipo de EM de acuerdo con los siguientes indicadores: i) síntomas musculares, ii) síntomas intestinales, iii) síntomas oculares. SistemaExperto_IUPB se encuentra registrado en Colombia, Dirección Nacional de Derecho de Autor, con número 13-34-113 del 27 de julio de 2012. El prototipo de sistema desarroIlado está accesible en la dirección:

http://www.itm.edu.co/trabajosdegradodestacados/index.html.

Es de interés efectuar otros estudios en los que se usen los SE en problemas que requieran inferencia determinista para hacer clasificaciones, por ejemplo de virus/antivirus, delitos, e intrusiones informáticas.

\section{AGRADECIMIENTOS}

Esta sección reconoce la asesoría de los expertos en esclerosis múltiple, pertenecientes al Instituto Neurológico de Antioquia. Este trabajo hace parte del grupo de investigación en Neurociencias de la Universidad CES y del grupo GIIEN de la Institución Universitaria Pascual Bravo -IUPB-.

\section{FINANCIAMIENTO}

Institución Universitaria Pascual Bravo

\section{REFERENCIAS}

AMERICAN ACADEMIC OF NEUROLOGY, C. (10 de 5 de 2011). Lifelong learning in neurology. Recuperado el 20 de 2 de 2012, de Lifelong learning in neurology: http://www.aan.com/elibrary/continuum/?event=home. .showlssue\&issue=ovid.com:/ issue/ovftdb/00132979-200412000-00000.

Astion, M., Wener, M., Thomas, R., Hunder, G., \& Bloch, D. (1994). Application of neural networks to the classification of giant cell arteritis. Arthritis and Rheumatism , 760-770.

Bartosh, A., Andersson, B., \& Nilsson, J. (2008). Artificial neural networks in pancreatic disease. British journal of surgery , 817-826.
Bizios, D., Heij, A., \& Bengtsson, B. (2007). Trained artificial neural network for galucoma diagnosis using visual field data: un comparison with conventional algorithms . Journal of glaucoma , 20-28.

Borges, K., Moura, R., \& Steiner, A. (2010). Diagnosis of headache using artificial neural network. IJCSNS International Journal of Computer Science and NetworkSecurity, 128-142.

Bourdes, V., Bonnevay, S., Bachelot, T., \& Perol, D. (2007). Breast cancer predictions by neural netwroks analysis: a comparison with logistic regression. Engineering in medicine and and biological society. 29th anuual international conference of the IEEE , 5424-5427.

Buarque, F. (2000). Multiple sclerosis plaque: computer model and simulations. Internal report. Imperial College of science technology and medicine, EEE Departure , 12, 51.

Celona, A., Grasso, G., \& Puccio, L. (2009). Artificial neural network. Morphological classification by euclidean distance histograms for prognostic evaluation of magnetic resonance imaging in multiple sclerosis. SIMAI Congress, 283-292.

Chiou, Y., \& Lure, Y. (1994). Hybrid lung module detection (HLND) system. Cancer Letters , 119-126.

Cook, D. (2005). Artificial neural network to predict mortality in critial care patients. An applocation of supervised machine learning. Australasian anaesthesia , 205-224.

Doyle, H., Dvorchik, I., Mitchell, S., Marino, I., Ebert, F., McMichael, J., y otros. (1994). Predicting outcomes after liver transplantations. A connectionist approach. Annals of surgery, 408-415.

Ebell, M. (1993). Artificial neural netwokrs for predicting failure to survive following hospital cardiopulmonary resuscitation. Journal of family practice, 297-303.

Esposito, M., \& De Pietro, G. (2011). An ontology-based fuzzy decision support system for multiple sclerosis. Elsevier. Engineering applications of artificial intelligence, 1340-1354.

Gannous, A., \& Elhaddad, R. (2011). Improving an artificial neural network model to predict thyroid bending protein diagnosis using preprocessing 
techniques. World academic of science, engineering and technology, 132-141.

Gaspari, M., Roveda, G., Scandellari, C., \& Stecchi, S. (2012). An expert system for the evaluation of EDSS in multiple sclerosis. Elsevier. Artificial intelligence in medicine, 187-210.

Giarratano, J., \& Riley, G. (2001). Sistemas experto. Principios y programacion. Mexico D.F.: International Thompson Editors.

Gil, D., Johnsson, M., Garcia, J., \& Soriano, A. (2009). Applications of artificial neural networks int the diagnosis of urological dysfunctions. Expert systems with applications, 5754-5760.

Gutierrez, A., Gonzalez, J., Lopez, P., Ojeda, E., Sanchez, J., \& Toro, J. (2001). Esclerosisi Multiple. Consensos en Neurologia: guias de practica clinica, 1-13.

Harrison, R., \& Kennedy, L. (2005). Artificial neural network models for prediction of acute coronary syndromes using clinical data from de time of presentation. Annals of emergency medicine. American collage of emergency, 228-251.

Kasmierczak, C., Catrou, G., \& Van Lente, F. (1998). Diagnostic accuracy of pancreatic enzymes evaluated by use of multivariate data analysis. Clinical chemestry, 817-826.
Primorac, C., \& Mariño, S. (4 de 12 de 2010). Un sistema experto para asistir decisiones turisticas. Diseño de un prototipo basado en la web. Recuperado el 9 de 5 de 1012, de Un sistema experto para asistir decisiones turisticas. Diseño de un prototipo basado en la web: http://www.eumed.net/rev/turydes/10/index.htm

Sharpe, P., Solberg, H., Rootwelt, K., \& Yearworth, M. (1993). Artificial neural networks in diagnosis of thyroid function from in vitro laboratory tests. Clinical chemestry, 2248-2253.

Shin, S., Tolum, M., \& Hassanpour, R. (2012). Hybrid expert system: A survey of current approachesand applications. Elsevier. Expert systems with applications , 4609-4617.

Tabares, H., Monsalve, D., \& Diez, D. (2013). Modelo de sistema experto para la seleccion de personal docente universitario. Tecnologicas , 35-54.

Wang, Y., \& Shiun, J. (2009). Artificial neural network to predict skeletal metastaisis in patients with prostate cancer. Spreniger science, Med Syst, 91-100.

Xu, C., Brown, D., Breit, S., \& Nakamura, T. (2006). Three new serum markers for prostate cancer detection within a percent free PSA-based artificial neural network. Prostate, 651-659. 